\title{
EDITORIAL
}

\section{Rational use of anti-tuberculosis drugs in the EU: better patient care and less drug resistance}

\author{
A. Zumla*, F. Blasi ${ }^{\#}$ and M. Raviglione
}

W orldwide, resistance to antibiotics routinely used to treat a range of common infectious diseases is increasing [1, 2]. Apart from microbial genetic factors, antimicrobial resistance (AMR) is generally attributed to widespread inappropriate antibiotic prescription and usage by physicians, poor drug quality and lack of adherence to proper treatment. The serious and ominous situation of AMR worldwide has led to the formulation and recent launch of the World Health Organization (WHO) policy package to combat AMR [3]. Resistance to tuberculosis (TB) drugs is of particular concern and has been highlighted recently [4-7]. Since the current portfolio of TB drugs is limited and drug-resistant TB is becoming widespread across all continents [8], the spread of drug-resistant TB now threatens the achievements made over a decade of intensive control efforts. TB drug resistance emerges spontaneously through chromosomal mutations in Mycobacterium tuberculosis strains. These can be induced by selection of mutants due to poor prescription, lack of patient adherence and poor drug quality [4-6]. The challenge of TB drug resistance extends beyond low-resource and high-TB-burden countries, now encompassing high-resource settings such as European Union (EU) member states. Worryingly, in the EU, resistance to first- and second-line TB drugs is failing to reduce and hot spots for drug-resistant TB within and beyond the EU borders remain [8-13]. Treatment for multidrug-resistant (MDR)-TB lasts at least 2 yrs, is very expensive to patients and society, is associated with several side-effects [14] and the treatment outcomes are comparatively poor $[7,15,16]$. Treatment is also challenging in the EU, with failure to reach acceptable levels of success rates particularly among MDR-TB patients [11].

Since the vast majority of the world's annual 9 million new cases of TB are drug sensitive [7, 17], the real challenge for the process of scaling up TB care and control, while preventing drug resistance, is to increase active and early case detection so that transmission can be interrupted by rapid start of treatment for both drug-susceptible and drug-resistant TB [18]. Optimal usage of current $\mathrm{TB}$ drugs, expanding access and preventing the development of drug resistance remain top priorities [19].

*Division of Infection and Immunity, Dept of Infection, University College London Medical School, London, UK. " Respiratory Medicine Section, Dipartimento Toraco-Polmonare e Cardiocircolatorio, University of Milan, IRCCS Fondazione Cà Granda, Milan, Italy. "World Health Organization, STOP TB Dept, Geneva, Switzerland.

CORRESPONDENCE: F. Blasi, Dipartimento Toraco-Polmonare e Cardiocircolatorio Università degli Studi di Milano, Milano, Italy. E-mail: francesco.blasi@unimi.it
The need for new TB drugs for treating drug-resistant $\mathrm{TB}$ is greater than ever and some progress is being made in new drug discovery and development, albeit slowly [20]. The new TB drug discovery pipeline appears promising; however, there are only two new classes of drugs, which are currently undergoing final phases of clinical trials and may be widely available in the next 2 yrs [19,21,22]. Recently, concerns have been raised about whether the use of new TB drugs should be prioritised for the treatment of drug-susceptible or drugresistant TB.

Therefore, it is critical that a comprehensive and universal approach to the optimal and rational use of old and new drugs for the treatment of drug-sensitive and drug-resistant TB is defined and developed jointly by clinicians, epidemiologists, scientists, drug developers, national programmes and policy makers. In support of this concept, the European Respiratory Journal (ERJ) will publish a series of five current state-of-the-art articles. These will define the correlation between inappropriate use of TB drugs and the emergence of drug-resistant $\mathrm{TB}$, supported by specific EU epidemiological and survey data.

In the current issue of the ERJ, the first article of the series, entitled "Prevalence of inappropriate tuberculosis treatment regimens: a systematic review", evaluates the prevalence and characteristics of inappropriate TB regimens as reported in the literature. Notwithstanding the lack of high quality data, the number and spectrum of inappropriate treatment regimens being used for TB in different geographical settings is highlighted [23].

The second article of the ERJ series, "Knowledge of tuberculosis treatment prescription of health workers: a systematic review", provides insights into the physician-related factors in the development of drug resistance [24].

The third article, "Multidrug resistance after inappropriate tuberculosis treatment: a meta-analysis", presents data from a systematic review that assessed the risk for TB drug resistance due to inappropriate TB regimens. This focuses on studies that utilised molecular typing methods to exclude re-infection and to confirm the association between the drug misuse and resistance. It concludes that inappropriate use of TB treatment regimens is the main cause of development of MDR-TB in the EU [25].

The fourth article is entitled "Protecting the new TB drugs pipeline: stating the case for the rational use of fluoroquinolones" by E. Huitric and co-workers. Fluoroquinolones (FQs) have the potential to become an important component of a 
future TB treatment regimen, and drugs like moxifloxacin, ofloxacin and gatifloxacin are currently being evaluated in phase III clinical trials. The introduction of these agents could greatly influence global TB control efforts. The current widespread use of FQs for treating community-acquired pneumonia and other respiratory infections, where TB may be undiagnosed or missed, is a real threat to adopting FQcontaining regimens. This article will describe the current existing guidelines in the EU on the use of FQs in respiratory infections. It will present data from a meta-analysis that shows that TB patients had a three-fold higher risk of developing FQ-resistant TB when prescribed FQs before TB diagnosis, compared with TB patients who were not exposed to FQs. The conclusion can be drawn that treatment with FQs before the diagnosis of $\mathrm{TB}$ is associated with a higher risk of developing FQ-resistant TB. This is an important consideration for chest physicians, since it is imperative that antibiotic prescribing practices are targeted towards preventing and managing AMR [6].

The fifth and final article is entitled "TB drug development and the potential to address the M/XDR-TB epidemic: relevance and implications for the rational use of drugs in the EU/EEA and beyond" by D. Manissero and co-workers. This will review the epidemiology of drug-resistant TB in the EU using available surveillance data. The promising new TB drug pipeline and ongoing clinical trials will be described, emphasising the need for more investment.

Numerous challenges exist to effectively contain TB drug resistance $[3,4,6,13,26]$. The WHO policy package to combat antimicrobial drug resistance addresses AMR in a broad sense, but all principles apply fully to TB drug resistance $[2,3]$. There are a number of factors that create the ideal conditions for irrational or inappropriate use of anti-TB drugs and they need to be tackled urgently. First, healthcare providers need to be trained using internationally recognised standards to avoid inappropriate prescription and practices [27]. Secondly, there is a general lack of legislation and its enforcement regulating use or dispensing of drugs, thus risking wrong or selfmedication. Thirdly, perverse economic incentives, such as profits to prescribers and pharmacists, may stimulate inappropriate prescription of drugs in the wrong doses and combinations. Potential solutions are available to address all these challenges and need to be systematically put into policy and practice to promote rational and judicious use of TB drugs.

The first set of measures is the promotion and enforcement of internationally recognised treatment and care guidelines that already exist [17] and are adopted in most programmatic settings worldwide among practitioners working for national programmes [1]. The challenge is to ensure that, in all healthcare facilities where TB care is delivered, health workers rigorously follow in routine practice the quality standards that national programmes promote on paper [28]. Importantly, in many countries today, TB drugs are prescribed by private practitioners and inadequate or irregular prescription due to the inability of the patients to buy the drugs and maintain continuity of treatment are leading to development of drug resistance [26]. Therefore, engagement of all health providers is essential, as promoted within the Stop TB Strategy [29]. In the EU, collaboration with professional societies like the European
Respiratory Society (ERS) and national societies, along with cooperation with the European Centers for Disease Control (ECDC) and WHO Regional Office for Europe may facilitate the process, especially when dealing with best prescribing practices $[27,28,30]$. In addition, collaboration is essential with health teaching institutions that train doctors, medical officers and nurses [31].

Inclusion of appropriate guidelines in the basic medical and paramedical curricula will be the key to prevention of drug resistance in the future [28]. Provision of continuous medical education for physicians and medical officers engaged in TB care will also inform best current practice. Finally, a crucial measure will be that of ensuring that a proper surveillance system does exist in all settings to monitor TB drug resistance levels and trends in the use of TB drugs.

The second set of measures consists in enforcing prescriptiononly use of anti-TB drugs. This may mean the establishment of effective legislation and licensing process for pharmacies where drugs are dispensed and clear rules limiting the availability of drugs to prescription-only categories. As part of these measures, there should be careful promotion of sale of proven-quality, combination drugs only, as the risk of developing resistance may increase when single drug treatment is used.

The third set of measures is about the promotion of education on the correct use of anti-TB drugs. The crucial step is to ensure that care providers and end users are fully aware of the proper and optimal way to use drugs and of the period of time necessary. The full engagement of patient groups and communities is another fundamental step to implement a strong policy in TB care and control.

Finally, the fourth set of measures is strongly policy oriented and focuses on reducing those financial incentives that encourage irrational use of medicines. The governments should also ensure that activities in the promotion of medicines are undertaken by industry according to well defined and enforced regulations. A code of good practice has been published by the International Federation of Pharmaceutical Manufacturers and Associations [32].

Ideally, these measures need to be coupled with the following: early diagnosis and treatment of active TB cases; support to, and observation of, treatment; systematic drug susceptibility testing; a continuous supply of good quality drugs; an effective recording and surveillance system; and constant audit of good prescribing policies. Tentatively in late 2012 or early 2013, two new compounds belonging to different and new classes of antiTB drugs will appear in the market [21, 22]. These recommendations on rational use of TB drugs will be even more important to ensure that these new TB drugs, against which resistance has not yet emerged, are preserved for future prolonged use by the millions of patients affected by TB and, especially, MDR-TB in the ensuing years. It is therefore imperative that all measures described here be put in place and a multidisciplinary effort is made to protect existing and future TB drugs.

This ERJ series of articles on drug-resistant TB in the EU is an important step forward in the synthesis of available information and in identifying gaps in knowledge and programmatic 
issues. It also reflects the important role and commitment required [33] from European organisations such as the ERS, the EU, ECDC and WHO in tackling, head on, TB drug resistance in the EU and globally.

\section{STATEMENT OF INTEREST}

None declared.

\section{REFERENCES}

1 Institute of Medicine (IOM) Forum on Microbial Threats. Antibiotic Resistance: Implications for Global Health and Novel Intervention Strategies - Workshop Summary. Washington, National Academies Press, 2010. Available from www.iom.edu/ Reports / 2010/Antibiotic-Resistance-Implications-for-GlobalHealth-and-Novel-Intervention-Strategies.aspx

2 World Health Organization. The Bulletin series: antimicrobial resistance. Available from www.who.int/bulletin/antimicrobial_ resistance/en/index.html Date last accessed: May 22, 2011.

3 Leung E, Weil DE, Raviglione M, et al. The WHO policy package to combat antimicrobial resistance. Bull World Health Organ 2011; 89: 390-392.

4 Yew WW. Management of multidrug-resistant tuberculosis and extensively drug-resistant tuberculosis: current status and future prospects. Kekkaku 2011; 86: 9-16.

5 Zignol M, van Gemert W, Falzon D, et al. Modernizing surveillance of antituberculosis drug resistance: from special surveys to routine testing. Clin Infect Dis 2011; 52: 901-906.

6 Raviglione MC, Lange C, Migliori GB. Preventing and managing antimicrobial resistance: imperative for chest physicians. Eur Respir J 2011; 37: 978-981.

7 Lawn S, Zumla AI. Tuberculosis. Lancet 2011; 378: 57-72.

8 Migliori GB, Sotgiu G, Lange C, et al. Extensively drug-resistant tuberculosis: back to the future. Eur Respir J 2010; 36: 475-477.

9 Manissero D, Hollo V, Huitric E, et al. Analysis of tuberculosis treatment outcomes in the European Union and European Economic Area: efforts needed towards optimal case management and control. Euro Surveill 2010; 15: 19514.

10 Hollo V, Zucs P, Ködmön C, et al. Marking 15 years of efforts towards a comprehensive European TB surveillance system: the epidemiological situation of TB in the EU/EEA in 2009. Euro Surveill 2011; 16: 19822.

11 European Centre for Disease Prevention and Control/WHO Regional Office for Europe. Tuberculosis Surveillance in Europe 2009. Stockholm, European Centre for Disease Prevention and Control, 2011.

12 World Health Organization. Multidrug and extensively drugresistant TB (M/XDR-TB): 2010 global report on surveillance and response. Publication No. WHO/HTM/TB/2010.3. Geneva, World Health Organization, 2010.

13 Fears R, Kaufmann S, Ter Meulen V, et al. Drug-resistant tuberculosis in the European Union: opportunities and challenges for control. Tuberculosis (Edinb) 2010; 90: 182-187.

14 Nathanson E, Gupta R, Huamani P, et al. Adverse events in the treatment of multidrug-resistant tuberculosis: results from the DOTS-Plus initiative. Int J Tuberc Lung Dis 2004; 8: 1382-1384.
15 Orenstein EW, Basu S, Shah NS, et al. Treatment outcomes among patients with multidrug-resistant tuberculosis: systematic review and meta-analysis. Lancet Infect Dis 2009; 9: 153-161.

16 Jacobson KR, Tierney DB, Jeon CY, et al. Treatment outcomes among patients with extensively drug-resistant tuberculosis: systematic review and meta-analysis. Clin Infect Dis 2010; 51: 6-14.

17 World Health Organization. Treatment of Tuberculosis: Guidelines. 4th Edn. Publication No. WHO/HTM/TB/2009.420. Geneva, World Health Organization, 2010.

18 Veen J, Migliori GB, Raviglione M, et al. Harmonisation of TB control in the WHO European region: the history of the Wolfheze Workshops. Eur Respir J 2011; 37: 950-959.

19 Yew WW, Lange C, Leung CC. Treatment of tuberculosis: update 2010. Eur Respir J 2011; 37: 441-462.

20 Mwaba P, McNerney R, Grobusch MP, et al. Achieving STOP TB Partnership goals: perspectives on development of new diagnostics, drugs and vaccines for tuberculosis. Trop Med Int Health 2011; [Epub ahead of print DOI: 10.1111/j.1365-3156.2011.02777.x].

21 Lienhardt C, Vernon A, Raviglione MC. New drugs and new regimens for the treatment of tuberculosis: review of the drug development pipeline and implications for national programmes. Curr Opin Pulm Med 2010; 16: 186-193.

22 Ma Z, Lienhardt C, McIlleron H, et al. Global tuberculosis drug development pipeline: the need and the reality. Lancet 2010; 375: 2100-2109.

23 Langendam MW, van der Werf MJ, Huitric E, et al. Prevalence of inappropriate tuberculosis treatment regimens: a systematic review. Eur Respir J 2012; 39: 1012-1020.

24 van der Werf MJ, Langendam MW, Huitric E, et al. Knowledge of tuberculosis treatment prescription of health workers: a systematic review. Eur Respir J 2012; [Epub ahead of print DOI: 10.1183/ 09031936.00125611].

25 van der Werf MJ, Langendam MW, Huitric E, et al. Multidrug resistance after inappropriate tuberculosis treatment: a metaanalysis. Eur Respir J 2012; [Epub ahead of print DOI: 10.1183/ 09031936.00125711].

26 Udwadia ZF, Pinto LM, Uplekar MW. Tuberculosis management by private practitioners in Mumbai, India: has anything changed in two decades? PLoS One 2010; 5: e12023.

27 Sotgiu G, Centis R, D'Ambrosio L, et al. Development of a standardised tool to survey MDR-/XDR-TB case management in Europe. Eur Respir J 2010; 36: 208-211.

28 Migliori GB, Sotgiu G, Blasi F, et al. Towards the development of EU/EEA Standards for Tuberculosis Care (ESTC). Eur Respir J 2011; 38: 493-495.

29 Raviglione MC, Uplekar MW. WHO's new Stop TB Strategy. Lancet 2006; 367: 952-955.

30 Sotgiu G, D'Ambrosio L, Centis R, et al. TB and M/XDR-TB infection control in European TB reference centres: the Achilles' heel? Eur Respir J 2011; 38: 1221-1223.

31 Eickelberg O, Laurent G, Nicod LP, et al. European Respiratory Society MD PhD programme in respiratory science. Eur Respir $J$ 2010; 36: 229-230.

32 International Federation of Pharmaceutical Manufacturers and Associations (IFPMA) Code of Pharmaceutical Marketing Practices - 2006 Revision. Geneva, IFPMA, 2006.

33 Fears R, Zumla A, ter Meulen V. European bodies can help to tackle TB worldwide. Nature 2009; 460: 796. 